\title{
On Wireless Power Transfer in Two-Tier Massive MIMO HetNets: Energy and Rate Analysis
}

\author{
Yongxu Zhu $\ddagger$ Lifeng Wang $\ddagger$ Kai-Kit Wong $\ddagger$ and Shi Jin ${ }^{\S}$ \\ ${ }_{\ddagger}^{\ddagger}$ Department of Electronic and Electrical Engineering, University College London, London, UK \\ $\S$ National Mobile Communications Research Laboratory, Southeast University, Nanjing 210096, China \\ e-mail: \{yongxu.zhu.13, lifeng.wang, kai-kit.wong\}@ucl.ac.uk, jinshi@seu.edu.cn
}

\begin{abstract}
In this paper, we investigate the potential application of wireless power transfer (WPT) in heterogeneous networks (HetNets) with massive multiple-input multiple-output (MIMO) antennas. Users first harvest energy from the downlink WPT, and then use the harvested energy for uplink transmission. We adopt the downlink received signal power (DRSP) based user association to maximize the harvested energy, and address the impact of massive MIMO on the user association. By using new statistical properties, we then obtain the exact expressions for the average harvested energy and the average uplink achievable rate of a user in such networks. Numerical results corroborate our analysis and demonstrate that compared to deploying more small cells, the use of a large number of antennas is more appealing since it brings in significant increase in the harvested energy of the HetNets. In addition, results illustrate that serving more users in the massive MIMO aided macrocells decreases the harvested energy and the uplink achievable rate of the HetNets.
\end{abstract}

\section{INTRODUCTION}

Traditional energy harvesting sources such as solar, wind, and hydroelectric power highly depend upon time and locations, as well as the conditions of the environments. Wireless power transfer (WPT) in contrast is a much more controllable approach to prolong the lifetime of mobile devices [1,2]. Recently, the potential of harvesting the ambient energy in the fifth-generation (5G) networks has been studied in [3].

Heterogeneous networks (HetNets) are identified as one of the key 5G enablers [4]. In HetNets, small cells are densely deployed [4], which shortens the distances between the mobile devices and the base stations (BSs). Recently, there is an interesting integration between WPT and HetNets, suggesting that stations, referred to as power beacons (PBs), be deployed in cellular networks for powering users via WPT [2].

Recent attempts have thus been to understand the feasibility of WPT in cellular networks. In particular, both picocell BSs and energy towers (or PBs) were considered in [5] to transfer energy to the users, and their problem was to jointly maximize the received energy and minimize the number of active picocell BSs and PBs. Subsequently in [6], user selection policies in dedicated RF-powered uplink cellular networks were investigated, where the BSs acted as dedicated power sources.

On the other hand, massive multiple-input multiple-output (MIMO) systems, using a large number of antennas at the BSs, promise an ultra-high spectral efficiency by accommodating a large number of users in the same radio channel. Their exceptional spatial selectivity also means that very sharp signal beams can be formed [7] and of great importance to WPT. Motivated by this, [8] studied the wireless information and power transfer in a point-to-point ( $\mathrm{P} 2 \mathrm{P})$ system including a single-antenna user and its serving BS equipped with a large antenna array, where energy efficiency for uplink information transfer was maximized under the quality-of-service $(\mathrm{QoS})$ constraint. In contrast to [8], [9] considered the uplink throughput optimization in a massive MIMO powered network, where an access point equipped with a large antenna array transfers energy to multiple users.

Regarded as a promising network architecture to meet the increasing demand for mobile data, massive MIMO empowered HetNets have recently attracted much attention $[10,11]$. Motivated by these efforts, this paper explores the potential benefits of massive MIMO HetNets for wireless power transfer (WPT) and wireless information transfer (WIT), which has not been well understood. To be specific, in this paper, we provide a tractable framework to examine the implementation of downlink WPT and uplink WIT in massive MIMO aided HetNets using a stochastic geometric model. Based on the downlink received signal power (DRSP) user association for maximizing the harvested energy, we derive the average harvested energy and the average uplink achievable rate in such networks. The results provide some important guidelines.

\section{Network DESCRIPTION}

We consider a two-tier time-division duplex (TDD) HetNet including macrocells and picocells. Each user first harvests the energy from its serving BS in the downlink, and uses the harvested energy for WIT in the uplink. Let $T$ be the duration of a communication block. The first and second sub-blocks of duration $\tau T$ and $(1-\tau) T$ are allocated to the downlink WPT and uplink WIT, respectively, where $\tau \in(0,1)$ is the time allocation factor. We assume that the first tier represents the class of macrocell BSs (MBSs), each of MBS is equipped with $N$ antenna array. The locations of the MBSs and picocell BSs (PBSs) are modelled by an independent homogeneous Poisson point process (HPPP) $\Phi_{\mathrm{M}}$ and $\Phi_{\mathrm{S}}$ with density $\lambda_{\mathrm{M}}$ and $\lambda_{\mathrm{S}}\left(\lambda_{\mathrm{M}} \geq \lambda_{\mathrm{S}}\right)$. In the macrocell, $S$ single-antenna users communicate with one MBS (assuming $N \gg S \geq 1$ ) in the uplink over the same time slot and frequency band, while in the picocell, only one single-antenna user is allowed to communicate with a single-antenna PBS at a time slot. We 
assume that perfect channel state information (CSI) is known at the BS, ${ }^{1}$ and universal frequency reuse is employed such that all of the tiers share the same bandwidth.

\section{A. User Association}

In this network, a user is associated with the BS based on the maximum DRSP. Considering the effect of massive MIMO, the average received power at a user that is connected with the $\ell$-th MBS $\left(\ell \in \Phi_{\mathrm{M}}\right)$ and the $j$-th PBS $\left(j \in \Phi_{\mathrm{S}}\right)$ in the PBS can be, respectively, expressed as

$$
P_{r, \ell}=G_{a} \frac{P_{\mathrm{M}}}{S} L\left(\left|X_{\ell, \mathrm{M}}\right|\right), P_{r, j}=P_{\mathrm{S}} L\left(\left|X_{j, \mathrm{~S}}\right|\right),
$$

where $G_{a}$ denotes the power gain (or array gain) obtained by the user associated with the MBS, $P_{\mathrm{M}}$ and $P_{\mathrm{S}}$ is the MBS's and PBS's transmit power, $L(|X|)=\beta|X|^{-\alpha}$ is the path loss function, $\beta$ is the frequency dependent constant value, $|X|$ denotes the distance, and $\alpha$ is the path loss exponent ( $\alpha=\alpha_{\mathrm{M}}$ for MBS and $\alpha=\alpha_{\mathrm{S}}$ for PBS).

The serving BS is selected for maximizing the average received power according to the following criterion:

$$
\text { BS : } \arg \max _{k \in\{\mathrm{M}, \mathrm{S}\}} P_{r, k}^{*},
$$

where $P_{r, \mathrm{M}}^{*}=\max _{\ell \in \Phi_{\mathrm{M}}} P_{r, \ell}$, and $P_{r, j}^{*}=\max _{j \in \Phi_{\mathrm{S}}} P_{r, j}$.

\section{B. Downlink WPT Model}

For wireless energy harvesting, the RF signals are interpreted as energy. Therefore, in the macrocell, we adopt the low-complexity linear maximum-ratio transmission (MRT) beamforming to transfer the power towards its $S$ intended users with equal-time sharing. ${ }^{2}$ The allocated time for power transfer for each intended user is $\frac{\tau T}{S}$. As the energy harvested from the noise is negligible, during the energy harvesting phase, the total harvested energy at a typical user $o$ that is associated with the MBS is given by

$$
\mathrm{E}_{o, \mathrm{M}}=\mathrm{E}_{o, \mathrm{M}}^{1}+\mathrm{E}_{o, \mathrm{M}}^{2}+\mathrm{E}_{o, \mathrm{M}}^{3},
$$

where $\quad \mathrm{E}_{o, \mathrm{M}}^{1}=\eta P_{\mathrm{M}} h_{o} L\left(\max \left\{\left|X_{o, \mathrm{M}}\right|, d\right\}\right) \frac{\tau T}{S}$ is the energy from the directed WPT, $\mathrm{E}_{o, \mathrm{M}}^{2}=$ $\eta P_{\mathrm{M}} h^{\prime}{ }_{o} L\left(\max \left\{\left|X_{o, \mathrm{M}}\right|, d\right\}\right) \frac{(S-1) \tau T}{S}$ is the energy from the isotropic WPT, and $\mathrm{E}_{o, \mathrm{M}}^{3} \stackrel{=}{=} \eta\left(I_{\mathrm{M}, 1} \tau T+I_{\mathrm{S}, 1} \times \tau T\right)$ is the energy from the ambient RF . Here, $0<\eta<1$ is the RF-to-DC conversion efficiency, $d$ denotes the reference distance, $h_{o} \sim \Gamma(N, 1)$ and $\left|X_{o, \mathrm{M}}\right|$ are, respectively, the small-scale fading channel power gain and the distance when the serving MBS recharges the typical user, and $h_{o}^{\prime} \sim \exp (1)$ is the small-scale fading channel power gain when the serving MBS directly transfers energy to other users in the same cell. In addition, we also have $I_{\mathrm{M}, 1}=\sum_{\ell \in \Phi_{\mathrm{M}} \backslash\{o\}} P_{\mathrm{M}} h_{\ell} L\left(\max \left\{\left|X_{\ell, \mathrm{M}}\right|, d\right\}\right)$ and $I_{\mathrm{S}, 1}=\sum_{j \in \Phi_{S}} P_{\mathrm{S}} h_{j} L\left(\max \left\{\left|X_{j, S}\right|, d\right\}\right) . I_{\mathrm{M}, 1}$ and $I_{\mathrm{S}, 1}$ are the

\footnotetext{
${ }^{1}$ In the practical TDD massive MIMO systems, the downlink CSI can be obtained through channel reciprocity based on uplink training [12].

${ }^{2}$ In this way, user receives the largest transferred power in a short time, which means that the user's battery can be quickly recharged.
}

sum of interference from the interfering MBSs and PBSs in the first tier, where $h_{\ell} \sim \Gamma(1,1)$ and $h_{j} \sim \exp (1)$ denote the small-scale fading interfering channel gain, $\left|X_{\ell, \mathrm{M}}\right|$ and $\left|X_{j, \mathrm{~S}}\right|$ denote the distance from a typical user to MBS $\ell \in \Phi_{\mathrm{M}} \backslash\{o\}$ (except the typical user's serving MBS) and PBS $j$.

The harvested energy at a typical user $O$ associated with the PBS can also be written as

$$
\mathrm{E}_{o, \mathrm{~S}}=\mathrm{E}_{o, \mathrm{~S}}^{1}+\mathrm{E}_{o, \mathrm{~S}}^{2},
$$

where $\mathrm{E}_{o, \mathrm{~S}}^{1}=\eta P_{\mathrm{S}} g_{o} L\left(\max \left\{\left|X_{o, \mathrm{~S}}\right|, d\right\}\right) \tau T$ is the energy from the isotropic WPT and $\mathrm{E}_{o, \mathrm{~S}}^{2}=\eta\left(I_{\mathrm{M}, \mathrm{S}}+I_{\mathrm{S}, \mathrm{S}}\right) \tau T$ is the energy from the ambient RF, $g_{o} \sim \Gamma(1,1)$ and $\left|X_{o, S}\right|$ are the small-scale fading channel power gain and the distance between a typical user and its associated MBS, respectively, and similar to the above, we also have $I_{\mathrm{M}, \mathrm{S}}=\sum_{\ell \in \Phi_{\mathrm{M}}} P_{\mathrm{M}} g_{\ell} L\left(\max \left\{\left|X_{\ell, \mathrm{M}}\right|, d\right\}\right), \quad I_{\mathrm{S}, \mathrm{S}}=$ $\sum_{P_{i}} P_{j, \mathrm{~S}} L\left(\max \left\{\left|X_{j, \mathrm{~S}}\right|, d\right\}\right)$, in which $g_{\ell} \sim \Gamma(1,1)$ $\sum_{j \in \Phi_{\mathrm{S}} \backslash\{0\}} P_{i}$

and $g_{j, \mathrm{~S}} \sim \Gamma(1,1)$ denote the small-scale fading interfering channel power gain, $\left|X_{\ell, \mathrm{M}}\right|$ and $\left|X_{j, \mathrm{~S}}\right|$ are the distance from a typical user to MBS $\ell$ and PBS $j \in \Phi_{\mathrm{S}} \backslash\{o\}$.

\section{Uplink WIT Model}

After energy harvesting, user $u_{i}$ transmits information signals to the serving BS with a specific transmit power $P_{u_{i}}$. In the uplink, each MBS uses linear zero-forcing beamforming (ZFBF) to simultaneously receive $S$ data streams from its $S$ intended users to cancel the intra-cell interference, which has been widely used in the massive MIMO literature [13-15].

For a typical user that is associated with its typical serving MBS, the received signal-to-interference-plus-noise ratio (SINR) at its typical serving MBS is given by

$$
\operatorname{SINR}_{\mathrm{M}}=\frac{P_{u_{o}} h_{o, \mathrm{M}} L\left(\max \left\{\left|X_{o, \mathrm{M}}\right|, d\right\}\right)}{I_{u, \mathrm{M}}+I_{u, \mathrm{~S}}+\delta^{2}},
$$

where $I_{u, \mathrm{M}}=\sum_{i \in \tilde{\mathcal{U}}_{\mathrm{M}} \backslash\{0\}} P_{u_{i}} h_{i} L\left(\max \left\{\left|X_{i}\right|, d\right\}\right), I_{u, \mathrm{~S}}=$ $\sum_{j \in \widetilde{\mathcal{U}}_{i}} P_{u_{j}} h_{j} L\left(\max \left\{\left|X_{j}\right|, d\right\}\right)$, and $h_{o, \mathrm{M}} \sim \Gamma(N-S+1,1)$ [15], $h_{i} \sim \exp (1), h_{j} \sim \exp (1)$ are the small-scale fading channel power gain, $\left|X_{o, \mathrm{M}}\right|,\left|X_{i}\right|,\left|X_{j}\right|$ are the distance between a typical user and its typical serving MBS, respectively, $\tilde{\mathcal{U}}_{\mathrm{M}}$ is the point process corresponding to the interfering users in the macrocells, while $\tilde{\mathcal{U}}_{i}$ is the point process corresponding to the interfering users in the $i$-th tier, and $\delta^{2}$ denotes the noise power.

Likewise, for a typical user associated with the typical serving PBS, the received SINR is given by

$$
\operatorname{SINR}_{\mathrm{S}}=\frac{P_{u_{o}} g_{o, \mathrm{~S}} L\left(\max \left\{\left|X_{o, \mathrm{~S}}\right|, d\right\}\right)}{I_{u, \mathrm{M}}+I_{u, \mathrm{~S}}+\delta^{2}},
$$

where $I_{u, \mathrm{M}}=\sum_{i \in \widetilde{\mathcal{U}}_{\mathrm{M}}} P_{u_{i}} g_{i} L\left(\max \left\{\left|X_{i}\right|, d\right\}\right), I_{u, \mathrm{~S}}=$ $\sum_{j \in \widetilde{\mathcal{U}}_{i} \backslash\{o\}} P_{u_{j}} g_{j} L\left(\max \left\{\left|X_{j}\right|, d\right\}\right), g_{o, \mathrm{~S}} \sim \exp (1), g_{i} \sim$ $\exp (1)$ and $g_{j} \sim \exp (1)$ are the small-scale fading channel gain, and $\left|X_{o, S}\right|,\left|X_{i}\right|$ and $\left|X_{j}\right|$ are the distance between the interfering user $u_{i}$ and the typical serving PBS, respectively. 


\section{ENERGY ANALYSIS}

Here, the average harvested energy is derived assuming that users are equipped with large energy storage so that users can transmit reliably after energy harvesting. Considering the fact that the energy consumed for uplink information transmission should not exceed the harvested energy, the stable transmit power $P_{u_{o}}$ for a typical user should satisfy [2]

$$
P_{u_{o}} \leq \overline{\mathrm{E}}_{o} /(1-\tau) T,
$$

where $\overline{\mathrm{E}}_{o}$ denotes the average harvested energy.

\section{A. New Statistical Properties}

Lemma 1. The probability density functions (PDFs) of the distance $\left|X_{o, \mathrm{M}}\right|$ and $\left|X_{o, \mathrm{~S}}\right|$ denote between a typical user and its serving $M B S$ and $P B S$, respectively, given by

$$
\begin{gathered}
f_{\left|X_{o, \mathrm{M}}\right|}(x)=\frac{2 \pi \lambda_{\mathrm{M}} x}{\Psi_{\mathrm{M}}} \exp \left(-\pi \lambda_{\mathrm{M}} x^{2}-\pi \lambda_{\mathrm{S}} \hat{r}_{\mathrm{MS}}^{2} x^{\frac{2 \alpha_{\mathrm{M}}}{\alpha_{\mathrm{S}}}}\right), \\
f_{\left|X_{o, S}\right|}(y)=\frac{2 \pi \lambda_{\mathrm{S}} y}{\Psi_{\mathrm{S}}} \exp \left(-\pi \lambda_{\mathrm{M}} \hat{r}_{\mathrm{SM}}^{2} y^{\frac{2 \alpha_{\mathrm{S}}}{\alpha_{\mathrm{M}}}}-\pi \lambda_{\mathrm{S}} y^{2}\right)
\end{gathered}
$$

in which $\hat{r}_{\mathrm{MS}}=\left(\frac{G_{a} P_{\mathrm{M}}}{S P_{\mathrm{S}}}\right)^{\frac{-1}{\alpha_{\mathrm{S}}}}$ with $G_{a}=(N+S-1)$, $\hat{r}_{\mathrm{SM}}=\left(\frac{S P_{\mathrm{S}}}{G_{a} P_{\mathrm{M}}}\right)^{\frac{-1}{\alpha_{\mathrm{M}}}}$. Also, in (8), $\Psi_{\mathrm{M}}$ is the probability that a typical user is associated with the MBS, given by $\Psi_{\mathrm{M}}=2 \pi \lambda_{\mathrm{M}} \int_{0}^{\infty} r \exp \left(-\pi \lambda_{\mathrm{M}} r^{2}-\pi \lambda_{\mathrm{S}} \hat{r}_{\mathrm{MS}}^{2} r^{\frac{2 \alpha_{\mathrm{M}}}{\alpha_{\mathrm{S}}}}\right) d r$, and $\Psi_{\mathrm{S}}$ is the probability that a typical user is associated with the PBS, which is given by $\Psi_{\mathrm{S}}=$ $2 \pi \lambda_{\mathrm{S}} \int_{0}^{\infty} r \exp \left(-\pi \lambda_{\mathrm{M}} \hat{r}_{\mathrm{SM}}^{2} r^{\frac{2 \alpha_{\mathrm{S}}}{\alpha_{\mathrm{M}}}}-\pi \lambda_{\mathrm{S}} r^{2}\right) d r$.

Proof. Based on the downlink WPT in Section II-B, the downlink received power gain is $G_{a}=(N+S-1)$, which is different from the conventional massive MIMO networks without energy harvesting, due to the fact that the interference is identified as an RF energy source.

Using the similar approach suggested by [16, Appendix A], we can then obtain the desired results (8) and (9).

\section{B. Average Harvested Energy}

In order to achieve the maximum average harvested energy can be achieved, we first derive the conditional expression of the average harvested energy given the distance between a typical user and its serving BS.

Theorem 1. Given the distances $\left|X_{o, \mathrm{M}}\right|=x$ and $\left|X_{o, \mathrm{~S}}\right|=y$, the conditional expressions of the average harvested energy for a typical user that is associated with an MBS and PBS, respectively, given by (10) and (11) at the top of next page, $d_{o}=\left(\hat{r}_{\mathrm{MS}}\right)^{-\frac{\alpha_{\mathrm{S}}}{\alpha_{\mathrm{M}}}} d^{\alpha_{\mathrm{S}} / \alpha_{\mathrm{M}}}$ and $d_{1}=\left(\hat{r}_{\mathrm{SM}}\right)^{\frac{-\alpha_{\mathrm{M}}}{\alpha_{\mathrm{S}}}} d^{\alpha_{\mathrm{M}} / \alpha_{\mathrm{S}}}$.

Proof. Based on (3), given $\left|X_{o, \mathrm{M}}\right|=x$, the average harvested energy for a typical user served by the MBS is written as

$$
\begin{aligned}
& \widetilde{\mathrm{E}}_{o, \mathrm{M}}(x)=\mathbb{E}\left\{\mathrm{E}_{o, \mathrm{M}}^{1}\right\}+\mathbb{E}\left\{\mathrm{E}_{o, \mathrm{M}}^{2}\right\}+\mathbb{E}\left\{\mathrm{E}_{o, \mathrm{M}}^{3}\right\} \\
& =\eta\left(\mathbb{E}\left\{I_{\mathrm{M}, 1}\right\}+\mathbb{E}\left\{I_{\mathrm{S}, 1}\right\}\right) \tau T+\eta G_{a} \frac{P_{\mathrm{M}}}{S} \beta \\
& \quad \times\left(\mathbf{1}(x \leq d) d^{-\alpha_{\mathrm{M}}}+\mathbf{1}(x>d) x^{-\alpha_{\mathrm{M}}}\right) \tau T .
\end{aligned}
$$

Here, $\mathbb{E}\left\{I_{\mathrm{M}, 1}\right\}$ is the average power harvested from the intra-tier interference, which is given by

$$
\begin{aligned}
& \mathbb{E}\left\{I_{\mathrm{M}, 1}\right\}=\mathbb{E}\left\{\sum_{\ell \in \Phi_{\mathrm{M}} \backslash\{o\}} P_{\mathrm{M}} h_{\ell} L\left(\max \left\{\left|X_{\ell, \mathrm{M}}\right|, d\right\}\right)\right\} \\
& \stackrel{(a)}{=} P_{\mathrm{M}} \beta 2 \pi \lambda_{\mathrm{M}}\left(\int_{x}^{\infty}(\max \{r, d\})^{-\alpha_{\mathrm{M}}} r d r\right) \\
& =P_{\mathrm{M}} \beta 2 \pi \lambda_{\mathrm{M}} \cdot \Xi\left(d, x, \alpha_{\mathrm{M}}\right),
\end{aligned}
$$

where $(a)$ is from $\mathbb{E}\left\{h_{\ell}\right\}=1$ and the Campbell's theorem,

$$
\begin{aligned}
\Xi\left(\delta, \zeta(x), \alpha_{o}\right) & =\mathbf{1}(x \leqslant \delta)\left(\left(d^{2}-\zeta(x)^{2}\right) /\left(2 d^{\alpha_{o}}\right)-d^{2-\alpha_{o}} /\left(2-\alpha_{o}\right)\right) \\
& -\mathbf{1}(x>\delta) \zeta(x)^{2-\alpha_{o}} /\left(2-\alpha_{o}\right) .
\end{aligned}
$$

Similarly, $\mathbb{E}\left\{I_{\mathrm{S}, 1}\right\}$ is the average power harvested from the inter-tier interference, which is given by

$$
\mathbb{E}\left\{I_{\mathrm{S}, 1}\right\}=P_{\mathrm{S}} \beta 2 \pi \lambda_{\mathrm{S}} \cdot \Xi\left(d_{o}, \hat{r}_{\mathrm{MS}}^{2} x^{\frac{2 \alpha_{\mathrm{M}}}{\alpha_{\mathrm{S}}}}, \alpha_{\mathrm{S}}\right),
$$

in which $d_{o}=\left(\hat{r}_{\mathrm{MS}}\right)^{-\frac{\alpha_{\mathrm{S}}}{\alpha_{\mathrm{M}}}} d^{\alpha_{\mathrm{S}} / \alpha_{\mathrm{M}}}$. By substituting (13) and (15) into (12), we then obtain (10).

Similarly, under a given distance $\left|X_{o, S}\right|=y$, the average harvested energy for a typical user served by the PBS can be derived as (11), where $d_{1}=\left(\hat{r}_{\mathrm{SM}}\right)^{\frac{-\alpha_{\mathrm{M}}}{\alpha_{\mathrm{S}}}} d^{\alpha_{\mathrm{M}} / \alpha_{\mathrm{S}}}$.

Overall, based on Theorem 1, for a user in the massive MIMO aided HetNets, the average harvested energy can be found as

$$
\begin{aligned}
\overline{\mathrm{E}}_{o, \text { HetNet }} & =\overline{\mathrm{E}}_{o, \mathrm{M}}+\overline{\mathrm{E}}_{o, \mathrm{~S}}=\Psi_{\mathrm{M}} \int_{0}^{\infty} \widetilde{\mathrm{E}}_{o, \mathrm{M}}(x) f_{\left|X_{o, \mathrm{M}}\right|}(x) d x \\
& +\Psi_{\mathrm{S}} \int_{0}^{\infty} \widetilde{\mathrm{E}}_{o, \mathrm{~S}}(y) f_{\left|X_{o, \mathrm{~S}}\right|}(y) d y
\end{aligned}
$$

\section{Uplink Performance EVAluation}

In this section, we analyze the uplink WIT performance in terms of the average achievable rate. We assume that each user intends to set the maximum stable transmit power to achieve the maximum achievable rate. Hence, the transmit power for user $i$ in a macrocell is $P_{u_{i}}=P_{u_{\mathrm{M}}}=\frac{\overline{\mathrm{E}}_{o, \mathrm{M}}}{(1-\tau) T}$, and the transmit power for user $j$ in a picocell is $P_{u_{j}}=P_{u_{\mathrm{S}}}=\frac{\overline{\mathrm{E}}_{o, \mathrm{~S}}}{(1-\tau) T}$, where $\overline{\mathrm{E}}_{o, \mathrm{M}}$ and $\overline{\mathrm{E}}_{o, \mathrm{~S}}$ are given by (16).

\section{A. Average Uplink Achievable Rate}

We present the achievable rate for the massive MIMO HetNet uplink have the following theorems.

Theorem 2. Given a distance $\left|X_{o, \mathrm{M}}\right|=x$, a tractable lower bound for the conditional average uplink achievable rate between a typical user and its serving MBS can be found as

$$
R_{\mathrm{M}}^{\text {low }}(x)=(1-\tau) \log _{2}\left(1+P_{u_{\mathrm{M}}}(N-S+1) \Delta_{1}(x) / \Lambda\right),
$$

where $\Delta_{1}(x)=\beta\left(\mathbf{1}(x \leq d) d^{-\alpha_{\mathrm{M}}}+\mathbf{1}(x>d) x^{-\alpha_{\mathrm{M}}}\right)$ and

$$
\Lambda=\left(P_{u_{\mathrm{M}}} S \lambda_{\mathrm{M}}+P_{u_{\mathrm{S}}} \lambda_{\mathrm{S}}\right) \frac{\pi \beta \alpha_{M} d^{2-\alpha_{M}}}{\alpha_{M}-2}+\delta^{2} .
$$




$$
\begin{aligned}
\widetilde{\mathrm{E}}_{o, \mathrm{M}}(x)=\eta \tau T\{( & \left.\mathbf{1}(x \leqslant d) \frac{P_{\mathrm{M}} \beta}{d^{\alpha_{\mathrm{M}}}}\left[\frac{G_{a}}{S}+\pi \lambda_{\mathrm{M}} \frac{\left(x^{2}-d^{2}\right) \alpha_{\mathrm{M}}-2 x^{2}}{2-\alpha_{\mathrm{M}}}\right]\right)+\left(\mathbf{1}(x>d) \frac{P_{\mathrm{M}} \beta}{x^{\alpha_{\mathrm{M}}}}\left[\frac{G_{a}}{S}-\frac{2 \pi \lambda_{\mathrm{M}} x^{2}}{2-\alpha_{\mathrm{M}}}\right]\right) \\
& \left.+P_{S} \beta 2 \pi \lambda_{\mathrm{S}}\left(\mathbf{1}\left(x \leqslant d_{o}\right)\left(\frac{\left(d^{2}-\hat{r}_{\mathrm{MS}}^{2} x^{\frac{2 \alpha_{\mathrm{M}}}{\alpha_{\mathrm{S}}}}\right)}{2 d^{\alpha_{\mathrm{S}}}}-\frac{d^{2-\alpha_{\mathrm{S}}}}{2-\alpha_{\mathrm{S}}}\right)-\mathbf{1}\left(x>d_{o}\right) \frac{\hat{r}_{\mathrm{MS}}^{\left(2-\alpha_{\mathrm{S}}\right)} x^{\frac{\alpha_{\mathrm{M}}\left(2-\alpha_{\mathrm{S}}\right)}{\alpha_{\mathrm{S}}}}}{2-\alpha_{\mathrm{S}}}\right)\right\},
\end{aligned}
$$

$$
\begin{aligned}
\widetilde{\mathrm{E}}_{o, \mathrm{~S}}(y)=\eta \tau T & \left\{\left(\mathbf{1}(y \leqslant d) \frac{P_{\mathrm{S}} \beta}{d^{\alpha_{\mathrm{S}}}}\left[1+\pi \lambda_{i} \frac{\alpha_{\mathrm{S}}\left(y^{2}-d^{2}\right)-2 y^{2}}{2-\alpha_{\mathrm{S}}}\right]\right)+\left(\mathbf{1}(y>d) \frac{P_{\mathrm{S}} \beta}{y^{\alpha_{\mathrm{S}}}}\left[1-\frac{2 \pi \lambda_{\mathrm{S}} y^{2}}{2-\alpha_{\mathrm{S}}}\right)\right\}\right. \\
+ & \left.P_{\mathrm{M}} \beta 2 \pi \lambda_{\mathrm{M}}\left(\mathbf{1}\left(y \leqslant d_{1}\right)\left(d^{-\alpha_{\mathrm{M}}} \frac{\left(d^{2}-\hat{r}_{\mathrm{SM}}^{2} y^{\frac{2 \alpha_{\mathrm{S}}}{\alpha_{\mathrm{M}}}}\right)}{2}-\frac{d^{2-\alpha_{\mathrm{M}}}}{2-\alpha_{\mathrm{M}}}\right)-\mathbf{1}\left(y>d_{1}\right) \frac{\hat{r}_{\mathrm{SM}}^{2-\alpha_{\mathrm{M}}} y^{\frac{\alpha_{\mathrm{S}}\left(2-\alpha_{\mathrm{M}}\right)}{\alpha_{\mathrm{M}}}}}{2-\alpha_{\mathrm{M}}}\right)\right\},
\end{aligned}
$$

Proof. The exact average achievable rate is written as

$$
R=[(1-\tau) T] / T \times \mathbb{E}\left\{\log _{2}(1+\mathrm{SINR})\right\} .
$$

Now, using Jensen's inequality, we can obtain the lower bound for the conditional average uplink achievable rate between a typical user and its serving MBS as

$$
R_{\mathrm{M}}^{\text {low }}(x)=(1-\tau) \log _{2}\left(1+1 / \mathrm{E}\left\{\operatorname{SINR}_{\mathrm{M}}^{-1}\right\}\right) .
$$

Based on (5), $\mathbb{E}\left\{\mathrm{SINR}_{\mathrm{M}}^{-1}\right\}$ is calculated as

$$
\begin{aligned}
& \mathbb{E}\left\{\mathrm{SINR}_{\mathrm{M}}^{-1}\right\}=\mathbb{E}\left\{\frac{I_{u, \mathrm{M}}+I_{u, \mathrm{~S}}+\delta^{2}}{P_{u_{\mathrm{M}}} h_{o, \mathrm{M}} L(\max \{x, d\})}\right\} \\
& \stackrel{(a)}{\approx} \frac{\mathbb{E}\left\{I_{u, \mathrm{M}}\right\}+\mathbb{E}\left\{I_{u, \mathrm{~S}}\right\}+\delta^{2}}{P_{u_{\mathrm{M}}}(N-S+1) L(\max \{x, d\})},
\end{aligned}
$$

where (a) is obtained by using the law of large numbers, i.e., $h_{o, \mathrm{M}} \approx N-S+1$ as $N$ becomes large. Using the Campbell's theorem, we next derive $\mathbb{E}\left\{I_{u, \mathrm{M}}\right\}$ as

$$
\begin{aligned}
& \mathbb{E}\left\{I_{u, \mathrm{M}}\right\}=P_{u_{\mathrm{M}}} \beta 2 \pi\left(S \lambda_{\mathrm{M}}\right)\left(\int_{0}^{d} d^{-\alpha_{\mathrm{M}}} r d r+\int_{d}^{\infty} r^{-\alpha_{\mathrm{M}}} r d r\right) \\
& =P_{u_{\mathrm{M}}} \beta 2 \pi\left(S \lambda_{\mathrm{M}}\right)\left(d^{2-\alpha_{\mathrm{M}}} / 2+d^{2-\alpha_{\mathrm{M}}} /\left(\alpha_{\mathrm{M}}-2\right)\right) .
\end{aligned}
$$

Likewise, $\mathbb{E}\left\{I_{u, \mathrm{~S}}\right\}$ is derived as

$$
\mathbb{E}\left\{I_{u, \mathrm{~S}}\right\}=P_{u_{\mathrm{S}}} \beta 2 \pi \lambda_{\mathrm{S}}\left(d^{2-\alpha_{\mathrm{M}}} / 2+d^{2-\alpha_{\mathrm{M}}} /\left(\alpha_{\mathrm{M}}-2\right)\right) .
$$

Substituting (21)-(23) into (20), we obtain (17).

Theorem 3. Given a distance $\left|X_{o, \mathrm{~S}}\right|=y$, the conditional average uplink achievable rate between a typical user and its serving $P B S$ is given by

$$
R_{\mathrm{S}}(y)=\frac{(1-\tau)}{\ln 2} \int_{0}^{\infty} \frac{\bar{F}_{\mathrm{SINR}_{\mathrm{S}}}(x)}{1+x} d x
$$

where $\quad \bar{F}_{\operatorname{SINR}_{\mathrm{S}}}(x)=e^{-\frac{x \delta^{2}}{P_{u_{\mathrm{S}}} \Delta_{2}(y)}-\Omega\left(\frac{x}{P_{u_{\mathrm{S}}} \Delta_{2}(y)}\right)}$ is the complementary cumulative distribution function (CCDF) of the received SINR, in which $\Delta_{2}(y)=\beta\left(\mathbf{1}(y \leq d) d^{-\alpha_{\mathrm{S}}}+\mathbf{1}(y>d) x^{-\alpha_{\mathrm{S}}}\right)$, and $\Omega(\cdot)$ is given by (25) (see next page). In (25), ${ }_{2} F_{1}[\cdot, \cdot ; \cdot ; \cdot \cdot]$ is the Gauss hypergeometric function.
Proof. In (24), $\bar{F}_{\mathrm{SINR}_{\mathrm{S}}}(x)$ is given by

$$
\begin{aligned}
\bar{F}_{\text {SINR }_{\mathrm{S}}}(x) & =e^{-\frac{x \delta^{2}}{P_{u_{\mathrm{S}}} \Delta_{2}(y)}} \mathbb{E}\left\{e^{-\frac{x I_{u, \mathrm{M}}}{P_{u_{\mathrm{S}}} \Delta_{2}(y)}}\right\} \mathbb{E}\left\{e^{-\frac{x I_{u, \mathrm{~S}}}{P_{u_{\mathrm{S}}} \Delta_{2}(y)}}\right\} \\
& =e^{-\frac{x \delta^{2}}{P_{u_{k}} \Delta_{2}(y)}} \mathcal{L}_{I_{u, \mathrm{M}}}\left(\frac{x}{P_{u_{\mathrm{S}}} \Delta_{2}(y)}\right) \mathcal{L}_{I_{u, \mathrm{~S}}}\left(\frac{x}{P_{u_{\mathrm{S}}} \Delta_{2}(y)}\right),
\end{aligned}
$$

where $\Delta_{2}(y)=L(\max \{y, d\}), \mathcal{L}_{I_{u, \mathrm{M}}}(\cdot)$ and $\mathcal{L}_{I_{u, \mathrm{~S}}}(\cdot)$ are the Laplace transforms of the PDFs of $I_{u, \mathrm{M}}$ and $I_{u, \mathrm{~S}}$, respectively:

$$
\begin{aligned}
& \mathcal{L}_{I_{u, \mathrm{M}}}(s)=\mathbb{E}\left\{\exp \left(-s \sum_{i \in \widetilde{\mathcal{U}}_{\mathrm{M}}} P_{u_{\mathrm{M}}} g_{i} L\left(\max \left\{\left|X_{i}\right|, d\right\}\right)\right)\right\} \\
& =\exp \left(-\pi\left(S \lambda_{\mathrm{M}}\right) s P_{u_{\mathrm{M}}} \beta\left(\frac{d^{-\alpha_{\mathrm{S}}}}{1+s P_{u_{\mathrm{M}}} \beta d^{-\alpha_{\mathrm{S}}}} d^{2}\right.\right. \\
& \left.\left.-\frac{2 d^{2-\alpha_{\mathrm{S}}}}{\alpha_{\mathrm{S}}-2} F_{2}\left[1, \frac{\alpha_{\mathrm{S}}-2}{\alpha_{\mathrm{S}}} ; 2-\frac{2}{\alpha_{\mathrm{S}}} ;-s P_{u_{\mathrm{M}}} \beta d^{-\alpha_{\mathrm{S}}}\right]\right)\right),
\end{aligned}
$$

Then, $\mathcal{L}_{I_{u, \mathrm{~S}}}(s)$ can be similarly obtained as (25) at the top of the following page.

Overall, with the help of Theorems 2 and 3, a lower bound on the average uplink achievable rate for a user in the massive MIMO aided HetNets with DRSP-based user association is calculated as

$$
\begin{aligned}
\bar{R}_{\text {HetNet }}^{\text {low }} & =\bar{R}_{\mathrm{M}}^{\text {low }}+\bar{R}_{\mathrm{S}}=\Psi_{\mathrm{M}} \int_{0}^{\infty} R_{\mathrm{M}}^{\text {low }}(x) f_{\left|X_{o, \mathrm{M}}\right|}(x) d x \\
& +\Psi_{\mathrm{S}} \int_{0}^{\infty} R_{\mathrm{S}}(y) f_{\left|X_{o, \mathrm{~S}}\right|}(y) d y
\end{aligned}
$$

\section{Numerical Results}

In this section, we present numerical results to examine the impact of different user association schemes and key system parameters on the harvested energy and the uplink achievable rate. The network is assumed to operate at carrier frequency $f_{c}=1 \mathrm{GHz}$; the bandwidth (BW) is $10 \mathrm{MHz}$, the density of MBSs and PBSs are $\lambda_{\mathrm{M}}=10^{-3}$ and $\lambda_{\mathrm{S}}$; the MBS's and PBS's 


$$
\begin{array}{r}
\mathcal{L}_{I_{u, \mathrm{~S}}}(s)=\pi\left(S \lambda_{\mathrm{M}}\right) \frac{s P_{u_{\mathrm{M}}} \beta d^{-\alpha_{\mathrm{S}}}}{1+s P_{u_{\mathrm{M}}} \beta d^{-\alpha_{\mathrm{S}}}} d^{2}+2 \pi\left(S \lambda_{\mathrm{M}}\right) s P_{u_{\mathrm{M}}} \beta \frac{d^{2-\alpha_{\mathrm{S}}}}{\alpha_{\mathrm{S}}-2}{ }_{2} F_{1}\left[1, \frac{\alpha_{\mathrm{S}}-2}{\alpha_{\mathrm{S}}} ; 2-\frac{2}{\alpha_{\mathrm{S}}} ;-s P_{u_{\mathrm{M}}} \beta d^{-\alpha_{\mathrm{S}}}\right] \\
+\pi \lambda_{\mathrm{S}} \frac{s P_{u_{\mathrm{S}}} \beta d^{-\alpha_{\mathrm{S}}}}{1+s P_{u_{\mathrm{S}}} \beta d^{-\alpha_{\mathrm{S}}}} d^{2}+2 \pi \lambda_{\mathrm{S}} s P_{u_{i}} \beta \frac{d^{2-\alpha_{\mathrm{S}}}}{\alpha_{\mathrm{S}}-2}{ }_{2} F_{1}\left[1, \frac{\alpha_{\mathrm{S}}-2}{\alpha_{\mathrm{S}}} ; 2-\frac{2}{\alpha_{\mathrm{S}}} ;-s P_{u_{\mathrm{S}}} \beta d^{-\alpha_{\mathrm{S}}}\right]
\end{array}
$$

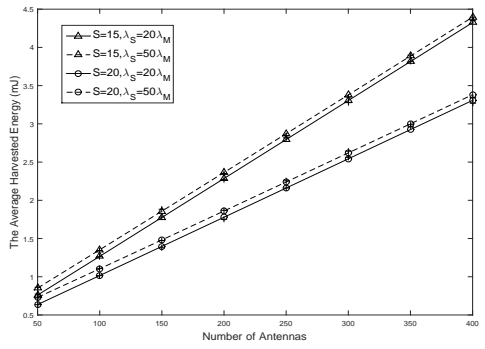

Fig. 1. Results for the average harvested energy: $T=1, \tau=0.7, \eta=0.8$, $\alpha_{\mathrm{M}}=2.5$ and $\alpha_{\mathrm{S}}=2.8$.

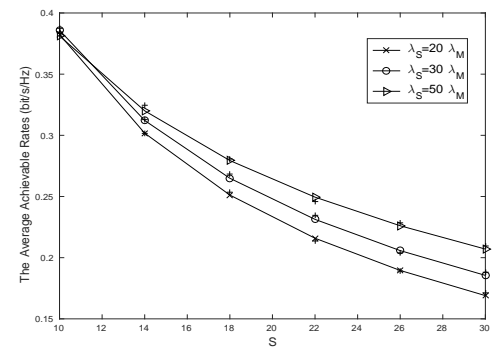

Fig. 2. Results for the average uplink achievable rate: $\tau=0.3, \eta=0.5$, $\alpha_{\mathrm{M}}=2.8$ and $\alpha_{\mathrm{S}}=2.5$, and $N=200$.

transmit power are $P_{\mathrm{M}}=46 \mathrm{dBm}$ and $P_{\mathrm{S}}=30 \mathrm{dBm}$; the noise figure is $\mathrm{Nf}=10 \mathrm{~dB}$, the noise power is $\sigma^{2}=-170+$ $10 \log _{10}(\mathrm{BW})+\mathrm{Nf}=-90 \mathrm{dBm}$; the frequency dependent value $\beta=\left(\frac{\mathrm{c}}{4 \pi f_{c}}\right)^{2}$ with $c=3 \times 10^{8} \mathrm{~m} / \mathrm{s}$. In the figures, Monte Carlo simulations are marked with ' + '.

Fig. 1 provides the results for the average harvested energy of a user in the massive MIMO HetNet. All the solid curves are obtained from (16). It is observed that adding more antennas at the MBS can substantially increase the harvested energy. In contrast, deploying more PBSs only results in a slight increase in the amount of harvested energy. Additionally, the harvested energy goes down when serving more uses in the macrocells because the directed power transfer time allocated to each user decreases with the number of users served by the MBS.

Fig. 2 demonstrates the results of the average uplink achievable rate for a user in the HetNet. The solid curves are obtained from (28). Results illustrate that serving more uses in the macrocells decreases the average uplink achievable rate for a user in the HetNet, because of the less harvested energy and more uplink interference. Moreover, deploying more PBSs can improve the uplink performance, since users are more close to the serving BSs.

\section{CONCLUSIONS}

In this paper, WPT was considered in the massive MIMO enabled HetNets. The downlink energy harvesting and uplink information transmission were evaluated in terms of the average harvested energy and the average achievable rate. Useful insights were obtained, providing helpful guidance.

\section{REFERENCES}

[1] R. Zhang and C. K. Ho, "MIMO broadcasting for simultaneous wireless information and power transfer,' IEEE Trans. Wireless Commun., vol. 12, no. 5, pp. 1989-2001, May 2013.

[2] K. Huang and V. K. N. Lau, "Enabling wireless power transfer in cellular networks: Architecture, modeling and deployment," IEEE Trans. Wireless Commun., vol. 13, no. 2, pp. 902-912, Feb. 2014.

[3] E. Hossain, M. Rasti, H. Tabassum, and A. Abdelnasser, "Evolution toward 5G multi-tier cellular wireless networks: An interference management perspective," IEEE Wireless Commun., vol. 21, no. 3, pp. 118127, June 2014

[4] J. Andrews, S. Buzzi, W. Choi, S. Hanly, A. Lozano, A. Soong, and J. Zhang, "What will 5G be?" IEEE J. Sel. Areas Commun., vol. 32, no. 6, pp. 1065-1082, Jun. 2014.

[5] M. Erol-Kantarci and H. Mouftah, "Radio-frequency-based wireless energy transfer in LTE-A heterogenous networks," in Computers and Communication (ISCC), 2014 IEEE Symposium on, June 2014, pp. 1-6.

[6] H. Tabassum, E. Hossain, M. Hossain, and D. Kim, "On the spectral efficiency of multiuser scheduling in RF-powered uplink cellular networks," IEEE Trans. Wireless Commun., vol. 14, no. 7, pp. 3586-3600, July 2015.

[7] H. Q. Ngo, E. G. Larsson, and T. L. Marzetta, "Energy and spectral efficiency of very large multiuser MIMO systems," IEEE Trans. Commun., vol. 61, no. 4, pp. 1436-1449, Apr. 2013.

[8] X. Chen, X. Wang, and X. Chen, "Energy-efficient optimization for wireless information and power transfer in large-scale MIMO systems employing energy beamforming," Wireless Communications Letters, IEEE, vol. 2, no. 6, pp. 667-670, Dec. 2013.

[9] G. Yang, C. K. Ho, R. Zhang, and Y. L. Guan, "Throughput optimization for massive MIMO systems powered by wireless energy transfer," IEEE J. Sel. Areas Commun., vol. 33, no. 8, pp. 1640-1650, Aug. 2015.

[10] $\mathrm{Y}$. $\mathrm{Xu}$ and $\mathrm{S}$. Mao, "User association in massive MIMO hetnets," CoRR, vol. abs/1501.03407, 2015. [Online]. Available: http://arxiv.org/abs/1501.03407

[11] E. Björnson, M. Kountouris, and M. Debbah, "Massive MIMO and small cells: Improving energy efficiency by optimal soft-cell coordination," in Proc. 2013 20th Int. Conf. on Telecommun., May 2013, pp. 1-5.

[12] T. L. Marzetta, "Noncooperative cellular wireless with unlimited numbers of base station antennas," IEEE Trans. Wireless Commun., vol. 9, no. 11 , pp. $3590-3600$, Nov. 2010.

[13] H. Huh, A. M. Tulino, and G. Caire, "Network MIMO with linear zeroforcing beamforming: Large system analysis, impact of channel estimation, and reduced-complexity scheduling," IEEE Trans. Inf. Theory, vol. 58, no. 5, pp. 2911-2934, May 2012.

[14] D. Bethanabhotla, O. Y. Bursalioglu, H. C. Papadopoulos, and G. Caire, "Optimal user-cell association for massive MIMO wireless networks," http://arxiv.org/abs/1407.6731, 2014.

[15] K. Hosseini, W. Yu, and R. S. Adve, "Large-scale MIMO versus network MIMO for multicell interference mitigation," IEEE J. Sel. Areas Commun., vol. 8, no. 5, pp. 930-941, Oct. 2014.

[16] H.-S. Jo, Y. J. Sang, P. Xia, and J. G. Andrews, "Heterogeneous cellular networks with flexible cell association: A comprehensive downlink SINR analysis," IEEE Trans. Wireless Commun., vol. 11, no. 10, pp. 3484 3495, Oct. 2012. 